\title{
CAMBIOS ESPACIO - TEMPORALES EN LA DIVERSIDAD DE LA COMUNIDAD DE AVES DE LAS LOMAS DE LACHAY LUEGO DEL EVENTO EL NIÑO 97 - 98
}

Claudia Véliz Rosas ${ }^{1}$, Edgar Sáchez $\operatorname{Infantas}^{1}$ Wendy Tori Maguiña ${ }^{1}$

\section{Resumen}

Ante la perturbación que constituyó el evento El Niño 97 -98 (ENSO), se evaluaron los cambios espacio - temporales en la diversidad de aves de las Lomas de Lachay como un paso previo al análisis del desarrollo de procesos de resiliencia. Se encontró que la diversidad de aves, describe un comportamiento post - ENSO, probablemente asociado a la baja productividad de la comunidad vegetal durante 1999. Los valores registrados para el año 2000 presentan un comportamiento similar al esperado durante un año normal. Por otro lado la diversidad muestra el paso de una distribución espacial homogénea durante los meses bajo el efecto del ENSO, hacia una distribución heterogénea durante los meses de la época seca de un año que tiende a ser normal. Se puede afirmar que la avifauna mediante los valores de diversidad que genera y la distribución espacial de la misma, desarrolla procesos de resiliencia que le han permitido mostrar el comportamiento que se espera ocurra bajo condiciones normales y un retorno a la estacionalidad característica del ecosistema.

Palabras clave: lomas, ENSO, perturbación, resiliencia

\begin{abstract}
Spatio - temporal changes in the avian diversity values from Lomas de Lachay, were evaluated in order to identify the development of resilience processes, after the ENSO 97 - 98 perturbation. The avian diversity, showed a post - ENSO process probably in association with the low herbaceous productivity during 1999. Values for 2000 year are similar to those expected for a normal year. Diversity showed an homogeneous spatial distribution during months with ENSO influence. This spatial distribution changed to an heterogeneous one during the dry months of a normal year. Results lead to the conclusion that birds, considering the diversity values and its spatial distribution, develop resilience processes that permit the bird community to show the expected values for a normal year, and a return to the characteristic seasonal changes in this ecosystem.
\end{abstract}

Keywords: lomas, ENSO, perturbation, resilienc

\section{Introducción}

La capacidad de un sistema, para regresar al estado que presentaba antes de ser sometido a una perturbación, es denominada resiliencia (Grimm \& Wissel, 1997). Un ecosistema puede estar sometido a varios tipos de perturbaciones, ya sean naturales o antropogénicas. Algunas perturbaciones naturales ocurren periódicamente, y muchas veces el ecosistema evoluciona con esta perturbación. Huston (1994) distingue el disturbio de la perturbación, siendo ésta última aquella que no necesariamente causa mortalidad, y desplazan alguna propiedad de la comunidad o del ecosistema, ya sea la entrada de nutrientes, biomasa, tasa de natalidad, etc. Un ejemplo de este tipo de perturbación fue la influencia del Evento El Niño 97 - 98 en las Lomas de Lachay.

En el Perú, el ecosistema de lomas se encuentra protegido sólo en la Reserva Nacional de Lachay (Lomas de Lachay) la cual pertenece al Sistema Nacional de Áreas Naturales Protegidas por el Estado (SINANPE). Este ecosistema presenta una marcada estacionalidad con la presencia de dos épocas: la época seca entre los meses de diciembre a abril, y la época húmeda entre junio y setiembre (Torres, 1981). La elevada humedad relativa y la baja evaporación aunada a otros factores fisiográficos, hacen que durante la época húmeda se forme una densa neblina, la cual al condensarse, favorece el desarrollo de la vegetación. Durante la época seca, sin embargo, la humedad relativa disminuye y la evaporación aumenta debido a la mayor incidencia de radiación solar. Así el principal factor limitante durante ésta época es la disponibilidad de agua (Brack, 1986). La vegetación que se desarrolla durante la época húmeda favorece la presencia de aves, insectos y mamíferos. Varios estudios (Cavareski, 1976; Sánchez, 1992; Delisle \& Savidge, 1997; Knick \& Rotenberry, 2000; Tellería et al., Sf.) confirman la directa relación existente entre la estructura vegetal y la diversidad de aves.

Durante el verano de 1998, el Evento El Niño trajo como consecuencia un incremento en los niveles de precipitación, permitiendo que la vegetación presente a finales de 1997 permanezca en la loma durante todo el verano de 1998, hasta empalmar con la época húmeda del mismo año. Estos cambios en la comunidad vegetal, influenciaron en la comunidad de

\footnotetext{
${ }^{1}$ Laboratorio de Ecología de Procesos, Dpto. de Biología, Universidad Nacional Agraria La Molina Lima - Perú. Av. La Universidad s/n. Apartado 456. Lima 100. Dirección electrónica: gaviotin@universia.edu.pe esi@lamolina.edu.pe
} 
aves, la cual mostró abundancias inusualmente elevadas, y aparición de especies poco frecuentes durante 1998 (Tori, 2000). El registro de abundancia de aves realizado durante 1998 e inicios de 1999 , fue continuado durante los años siguientes.

Este trabajo tiene como objetivo evaluar los cambios espacio - temporales de la diversidad de aves en las Lomas de Lachay, como una evidencia de la existencia de resiliencia.

\section{Métodos}

La Reserva Nacional de Lachay, se encuentra a $105 \mathrm{Km}$. al norte de la ciudad de Lima - Perú

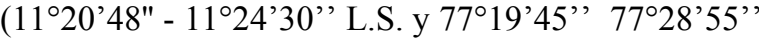
de L.O). El estudio se realizó en un área de $430 \mathrm{Ha}$. en donde se realizó un muestreo sistemático ubicándose un total de 31 parcelas. En cada parcela se realizó un conteo por puntos (Bibby et al., 1993) de una duración de 20 minutos, tiempo estandarizado por Tori (2000). Se registró la abundancia de cada especie avistada 30 metros a la redonda, calculándose luego la diversidad para cada parcela mediante el índice de Shannon Wienner (H) (Magurran ,1988):

$\mathrm{H}=-\Sigma\left(\mathrm{p}_{\mathrm{i}} \log _{2} \mathrm{p}_{\mathrm{i}}\right)$

Donde, $\mathrm{p}_{\mathrm{i}}=$ proporción de individuos

pertenecientes a la $i$-esima especie.

Se tomaron datos cuatro veces al año, dos veces durante la época seca, y dos durante la época húmeda. Así se obtuvieron registros para los meses de setiembre y noviembre de 1999; febrero, junio, agosto y diciembre del 2000; febrero, setiembre y diciembre del 2001; y junio del 2002. Los datos para junio 2001 y febrero del 2002 no se pudieron obtener por presentarse dificultades logísticas. Para el análisis se incluyeron datos obtenidos por Tori (2000) durante 1998 e inicios de 1999.

Para el análisis espacial y sus cambio a través del tiempo, se realizó un diagrama de dispersión entre la diversidad promedio mensual y su coeficiente de variabilidad espacial $(\mathrm{CV})$. El análisis de los cambios temporales de la diversdiad, se llevó a cabo mediante el uso de un ANVA de medias repetidas tomando como casos las parcelas y como tratamiento a los meses de muestreo. La prueba de contrastes de Tkey, su usó para detectar diferencias entre años y meses.

\section{Resultados}

La diversidad se distribuyó de manera homogénea (bajo CV) en toda el área de muestreo durante los meses que registraron valores altos de diversidad promedio. Por otro lado, ésta distribución fue heterogénea (alto CV) durante los meses con diversidad promedio baja. (Figura 1).

La diversidad promedio fue diferente entre los distintos meses $(\mathrm{F}=9.192, \mathrm{p}=0.0001)$. Durante 1998 la diversidad fue mayor a los otros tres años de estudio. Entre éstos últimos tres años la diversidad fue similar (Tabla 1). De hecho se observa en la Figura 2 que la diversidad promedio mensual fue poco variable durante 1998 registrando los valores más altos de toda la serie.

A nivel de los meses de febrero se observan diferencias antes y después del año 2000, ya que la diversidad de febrero de 1998 fue similar a la de febrero de $1999(\mathrm{p}=0.238)$, y ambas diferentes $y$ mayores a las obtenidas durante los meses de febrero de los años siguientes. (Figura 1(a)). Por su parte el análisis de los meses de mayo/junio mostró que la diversidad fue diferente y mayor durante 1998, mientras que entre 1999 y el año 2000 no hubieron diferencias $(p=0.769)$. Junio del 2001 muestra un comportamiento intermedio (Figura 1 (b)). En cuanto a los meses de agosto/setiembre, se obtuvo que sólo setiembre 1999 fue diferente de los otros meses análogos, los cuales fueron similares entre sí. (Figura 1 (c)). Por último diciembre 1998 fue distinto y mayor a sus meses análogos de años posteriores $(99,00$ y 01$)$ siendo éstos últimos meses similares entre sí (Figura 1 (d)). Se observa entonces que la tendencia general es mostrar diversidades mayores durante los meses del año 1998 e inicios de 1999 (febrero 1999).

Tabla 1. Valores de probabilidad de la prueba Tukey para los diferentes años.

\begin{tabular}{|c|c|c|c|c|}
\cline { 2 - 5 } \multicolumn{1}{c|}{} & 1998 & 1999 & 2000 & 2001 \\
\hline 19 & -- & 0.0001 & 0.0001 & 0.0001 \\
98 & & $4^{*}$ & $4^{*}$ & $5^{*}$ \\
\hline 19 & 0.0001 & -- & 0.9923 & 0.9827 \\
99 & $4^{*}$ & & 9 & 4 \\
\hline 20 & 0.0001 & 0.9923 & -- & 0.9156 \\
00 & $4^{*}$ & 9 & & 8 \\
\hline 20 & 0.0001 & 0.9827 & 0.9156 & -- \\
01 & $5^{*}$ & 4 & 8 & \\
\hline
\end{tabular}

\section{Discusión}

El análisis espacio - temporal muestra un evidente cambio en la distribución espacial de la diversidad a medida que se aleja del ENSO. Dentro de los meses con mayor diversidad y homogeneidad espacial (bajo $\mathrm{CV})$ se encuentran los cinco primeros meses, los cuales estuvieron bajo los efectos del ENSO. Uno de los efectos de esta perturbación, sería entonces homogenizar la distribución de la diversidad en toda la loma. Cabe resaltar que en este grupo se encuentra también setiembre del 2000, único mes de este año con diversidad alta y homogeneamente distribuída en el espacio. Esto sugiere que éste año se comporta como un año normal, ya que usualmente setiembre es el mes de mayor apogeo de las lomas. Los otros meses se agrupan hacia el otro extremo, (menor diversidad y mayor variabilidad espacial) dando a entender que cuanto mas lejos del ENSO, la diversidad ha ido disminuyendo retrayéndose en ciertos lugares, lo cual origina una mayor variabilidad espacial. Esto denota 
una recuperación de la estacionalidad característica del ecosistema, ya que en este grupo se encuentran febrero 2000 y febrero 2001, meses típicamente secos y donde se espera que la diversidad sea menor y se concentre sólo en algunos lugares. Es interesante el agrupamiento que muestra la diversidad según sus valores y distribución en el espacio, ya que denota que la comunidad de aves refleja dos estados bien diferenciados, influenciados indirectamente por los cambios en las condiciones ambientales que el ENSO generó durante 1998.

Para comprobar la ocurrencia de tales cambios se hizo el análisis temporal al interior de cada mes. Los resultados para mayo/junio y noviembre/diciembre, sugieren que tales diferencias aparecen a partir de junio 1999. No obstante, para los meses de agosto/setiembre, se esperaría que el único mes diferente y con mayor diversidad sea agosto 1998 y que los demás se parezcan entre sí. Sin embargo, dado que uno de los efectos del ENSO fue alargar la época húmeda del año 1997 empalmando con la del año 98, se podría esperar que la diversidad durante los siguientes meses de setiembre de un supuesto año normal sean similares o algo menores a los valores obtenidos durante el último ENSO. Ésto no deja de ser una especulación, pues no se cuentan con datos antes del ENSO, pero lleva a cuestionar el porqué entonces, la diversidad para setiembre 1999 no se parece a las otras tres, si el supuesto "retorno" a "condiciones normales" se daría a partir de junio 1999. Además la diversidad de setiembre 1999 resulta ser la menor de toda la serie, lo que llama la atención tratándose del mes que durante un año normal registra valores más altos.

La prueba estadística detecta que durante 1998 la diversidad fue mayor que en los otros años, y no se detectan diferencia alguna entre 1999 y el año 2000, diferencias que si se observan a nivel gráfico. En la figura 2 se observa, que durante 1999 la diversidad cae alcanzando su valor mas bajo en setiembre. Es cierto que ésta caída denota el inicio de un proceso distinto al ocurrido durante el ENSO, pero no del retorno a supuestas condiciones normales. Es sólo durante el año 2000, que la curva describe un comportamiento parecido al que se esperaría durante un año normal, con la más alta diversidad para el mes de setiembre y valores bajos y medios para el resto del año. Algo similar ocurre para el 2001. El proceso post - ENSO de 1999, caracterizado por la caída en la diversidad, podría estar relacionado con la disponibilidad de recursos. En efecto, Arana et. al. (1999) reporta para las Lomas de Lachay, que durante el año 1999, la vegetación fue escasa en comparación a otros años debido a que la humedad en las lomas disminuyó ocasionando una disminución en la abundancia de especies herbáceas. Ya que las aves granívoras son un grupo importante en la comunidad de aves de las Lomas de Lachay (Véliz, 2002) es de esperarse que se hayan visto afectadas por éstos cambios, y que esto se traduzca en una caída en los valores de diversidad. Un proceso similar se ha reportado tanto para las plantas como para los mamíferos de la isla Barro Colorado en Panamá (Wright, 2001), en donde la época seca estimula la floración en las plantas. La severa estación seca durante el ENSO 97-98 estimuló una sobreproducción de frutos. Al siguiente año una estación seca suave, provocó al parecer una caída en la producción de frutos, lo que ocasionó hambrunas entre los mamíferos granívoros y frugívoros. La hipótesis que plantea Wright (2001), es que el ENSO origina condiciones ambientales que favorecen una alta producción de frutos, producción que consume los recursos energéticos guardados por las plantas. Esto aunado a las condiciones ambientales, reduce la producción de frutos al año siguiente, lo cual repercute en los mamíferos que dependen de éstos recursos. Esta explicación, aunque aplicada a un ecosistema distinto, puede dar luces sobre lo que ocurre durante 1999 con la vegetación y la avifauna de las Lomas de Lachay. Esta comunidad de aves estaría desarrollando un proceso post-ENSO antes de mostrar el comportamiento que se espera durante un año normal.

\section{Conclusiones}

- El paso de una distribución espacial homogénea de la diversidad hacia una agregada durante la época seca de los últimos años, sugiere que el ecosistema recupera su estacionalidad apoyando la hipótesis del desarrollo de procesos de resiliencia.

- Las diferencias en la diversidad a partir de junio de 1999, están relacionadas con la baja productividad de la comunidad vegetal y forman parte de un proceso post-ENSO, previo al retorno a condiciones normales.

- El comportamiento de la diversidad durante el año 2000 sugiere que los efectos perturbadores del ENSO sobre la avifauna dejan de manifestarse, lo cual permite afirmar que existen de procesos de resiliencia en la comunidad de aves.

\section{Agradecimientos}

Éste trabajo fue posible gracias al financiamiento otorgado por CONCYTEC en el marco de la Red de Impacto Biológico "El Niño" RIBEN. Asímismo agradecemos el apoyo logístico recibido por alumnos y egresados de la carrera de biología de la UNALM, en la toma de datos. De manera especial a Carlos Alberto Arnillas y Carolina Tovar por sus valiosas sugerencias.

\section{Referencias Bibliográficas}

Arana C., Cano A., Roque J. y La Torre M. 1999. Influencia de los eventos "El Niño" y "La Niña" sobre la dominancia de especies de plantas herbáceas de las Lomas de Lachay. En: Reporte Lachay. Huaura - Perú 2000. Ministerio de 
Agricultura. Instituto Nacional de Recursos Naturales. Lima - Perú. p. 9

Bibby C., Burgess N. \& Hill D. 1993. Bird Census Techniques. Academic Press. Harcourt Brace \& Company, Publishers. London.

Brack A. 1986. La fauna del desierto costero. En: Gran Geografía del Perú. (3): 65-87.

Cavareski C.A. 1976. Relation of park size an vegetation to urban bird populations in Seattle, Washington. The Condor. 78: 375-382.

Delisle J.M. \& Savidge J.A. 1997. Avian use and vegetation characteristics of conservation reserve program fields. J. Wildl. Manage. 61(2): 318-325.

Grimm V. \& WisseL C. 1997. Babel, or the ecological stability discussions: an inventory and analysis of terminology and a guide for avoiding confusion. Oecologia. 109: 323-334.

Knick S.T. \& Rotenberry J.T. 2000. Ghosts of habitats past: Contribution of landscape change to current habitats used by shrubland birds. Ecology. 81 (1): 220-227.

Huston M. 1994. Biological Diversity The coexistence of species on Changing landscapes. Cambridge University Press N.Y. : 215-216.
Sánchez E. 1992. Diversidad y Estabilidad de Comunidades de Aves en la Reserva Nacional de Lachay. Tesis de Magister Scientiae. Universidad Nacional Agraria La Molina, Lima. Perú.

Tellería J.L., Santos T, Alvarez G. y Sáez-Royuela C. Sf. Avifauna de los campos de cereales del interior de España. Sociedad española de Ornitología. España.

Tori W. 2000. Análisis de los patrones de variación espacio-temporal de las poblaciones de Aves de la Reserva Nacional de Lachay, durante 1998-1999. Tesis para optar el Título de Bióloga, Universidad Nacional Agraria La Molina, Lima. Perú.

Torres J. 1981. Productividad Primaria en las Lomas de la Costa central del Perú. Boletín de Lima, $\mathrm{N}^{\mathrm{o}}$ 14. Lima. Perú.

Veliz C. 2002. Resiliencia en comunidades de aves de las Lomas de Lachay luego del Evento El Niño 9798. Tesis para optar el Título de Bióloga, Universidad Nacional Agraria La Molina, Lima. Perú.

Wright S. J. 2001. El fenómeno de El Niño y los bosques de isla Barro Colorado. En: S. HeckadonMoreno, editor. Panamá: Puente biológico. Imprelibros, S. A., Colombia. : 112 - 116.

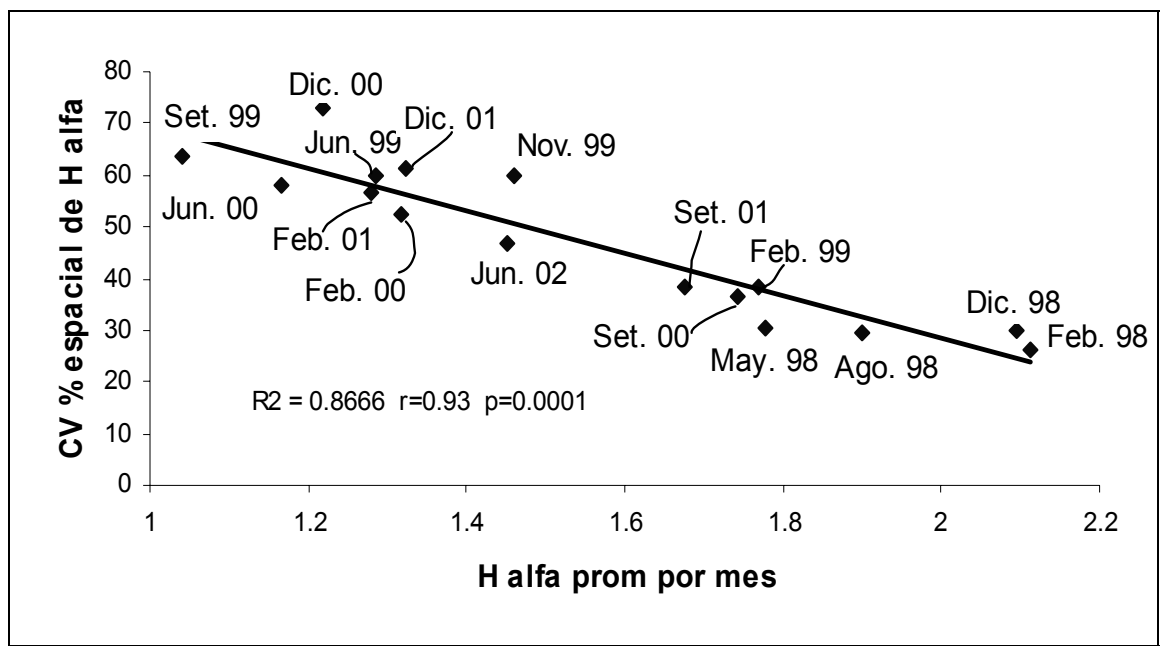

Figura 1. Diagrama de dispersión entre la diversidad alfa promedio mensual y su coeficiente de variabilidad espacial. 


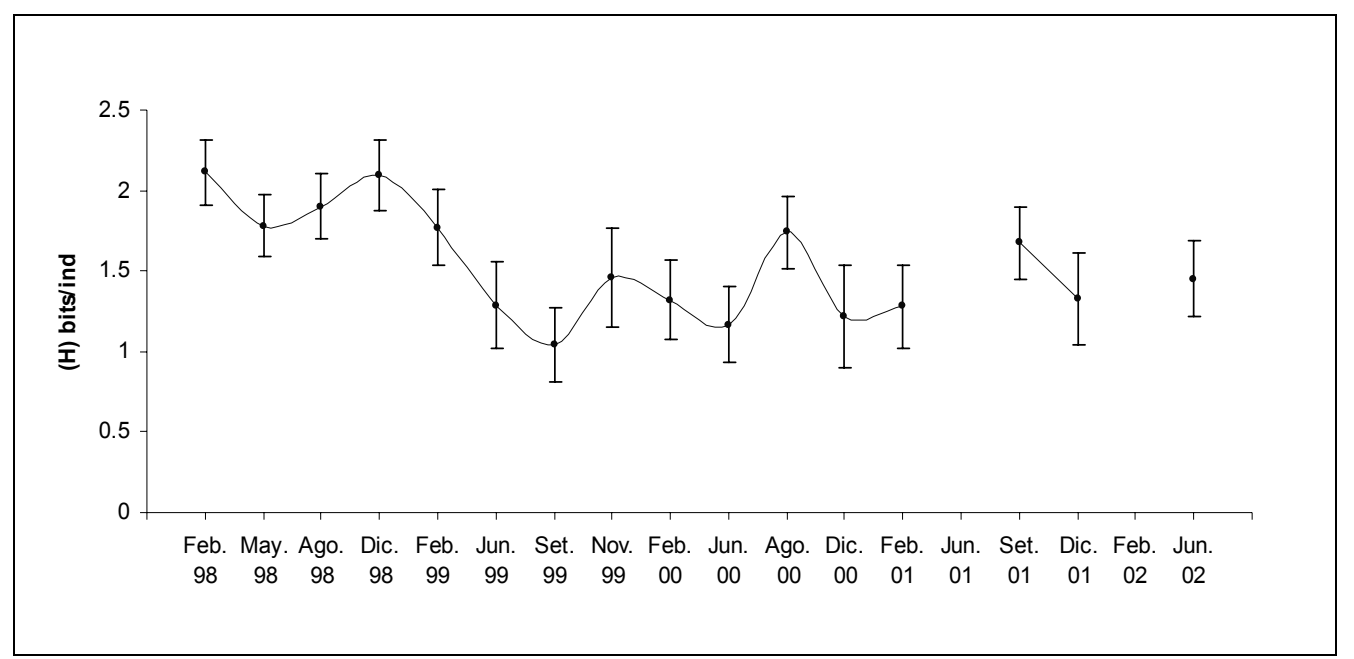

Figura 2. Valores de diversidad alfa a través del tiempo y sus intervalos de confianza.

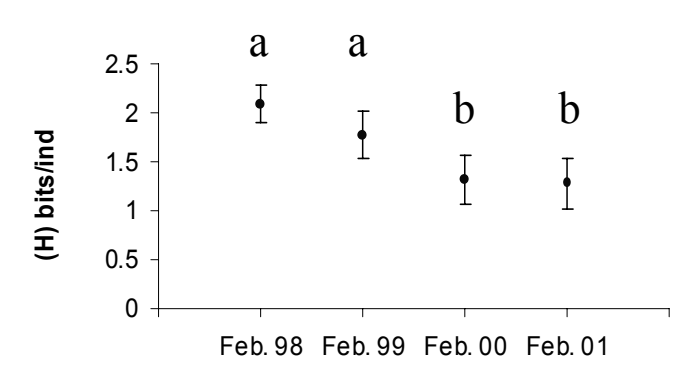

(a)

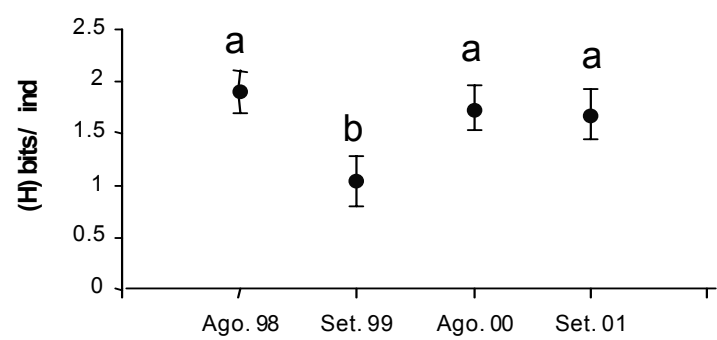

(c)

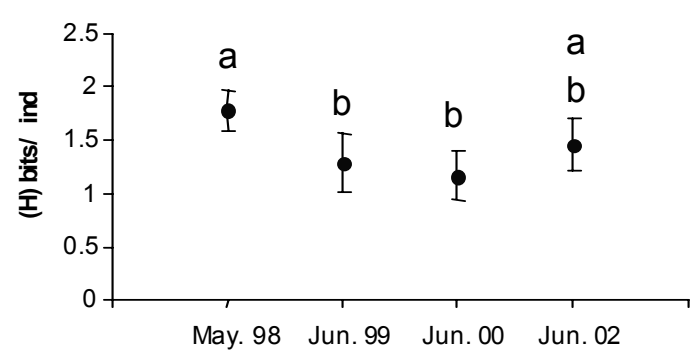

(b)

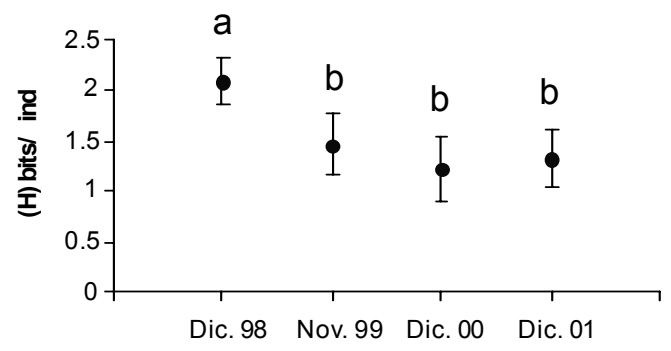

(d)

Figura 3. Valores de diversidad alfa e intervalos de confianza para los meses de febrero (a) mayo/junio (b) agosto/setiembre (c) y noviembre/diciembre (d). 